\title{
INFECÇÕES PARASITÁRIAS DO APÊNDICE CECAL E SUAS RELAÇÕES COM APENDICITE AGUDA
}

\author{
Danielle Fernandes da SILVA1', Reinaldo José da SILVA', Márcia Guimarães da SILVA², \\ Alesso Cervantes SARTORELLI², Bonifácio Katsunori TAKEGAWA ${ }^{3}$, \\ Maria Aparecida Marchesan RODRIGUES²
}

\begin{abstract}
RESUMO - Investigou-se a prevalência de infecções parasitárias do apêndice cecal e suas relações com a apendicite. Dos 1.600 apêndices estudados 24 (1,5\%) apresentaram infecção parasitária. Enterobius vermicularis foi encontrado em 23 casos $(95,8 \%)$ e Taenia sp em apenas um (4,2\%). Dezesseis pacientes (66,7\%) eram menores de 10 anos; 15 eram masculinos e 9 femininos. A análise histopatológica demonstrou inflamação aguda supurativa em 12 casos (50\%), eosinofilia em 13 (54,2\%) e hiperplasia linfóide em 10 (41,7\%). Complicações como peritonite ocorreram em 11 e gangrena em 3 casos. As infecções parasitárias do apêndice são causa pouco freqüente de apendicite aguda em crianças e adolescentes.
\end{abstract}

DESCRITORES - Apêndice. Doenças do ceco. Doenças parasitárias. Apendicite.

\section{INTRODUÇÃO}

As infecções parasitárias intestinais são eventos comuns em nosso meio e ocorrem em todas as faixas etárias e níveis socioeconômicos. Podem se expressar por manifestações clínicas atípicas, simulando quadros de apendicite ${ }^{(1,3,4,5)}$. Enterobius vermicularis, Ascaris sp, Schistosoma sp e, raramente, Taenia sp têm sido identificados em casos de apendicite aguda ${ }^{(2,4)}$. A presença de helmintos ao exame histopatológico do apêndice cecal tem despertado questionamentos sobre seu possível papel na gênese da apendicite.

Investigou-se no presente estudo a prevalência de infecções parasitárias do apêndice cecal e suas possíveis relações com a apendicite aguda.

\section{MÉTODOS}

Foram estudados retrospectivamente 1.600 apêndices cecais removidos cirurgicamente, com diagnóstico clínico de apendicite, no Hospital das Clínicas da Faculdade de Medicina de Botucatu - UNESP, Botucatu, SP, entre 1995 e 2005. Todos os apêndices foram submetidos a exame anatomopatológico. Para este procedimento foram fixados em solução de formalina a 10\%, seccionados em um corte longitudinal e dois cortes transversais, e processados para obtenção de cortes histológicos, que foram corados pela hematoxilina-eosina e analisados em microscópio de luz, Zeiss. As informações dos pacientes, tais como dados demográficos e quadro clínico, foram obtidas através de revisão dos prontuários.

\section{RESULTADOS}

Dos 1.600 apêndices cecais estudados, 24 apresentaram infecção parasitária. $\mathrm{O}$ helminto identificado com maior freqüência foi o $E$. vermicularis, presente em 23 dos 24 casos (95,8\%). Proglotes de Taenia sp. foram encontrados na luz do apêndice em único caso $(4,2 \%)$. Os dados demográficos dos pacientes são apresentados na Tabela 1 . Dezesseis pacientes $(66,7 \%)$ eram menores de 10 anos; 15 eram masculinos e 9 femininos; 21 pacientes eram brancos e 3 não-brancos. Todos referiam alterações do hábito intestinal e apresentavam sintomas de dor abdominal, sendo que em nove casos $(37,5 \%)$ havia irradiação da dor para a fossa ilíaca direita. O estudo histopatológico dos apêndices com infecção parasitária demonstrou processo inflamatório agudo supurativo, que dissociava as camadas musculares em 11 dos 24 casos $(45,8 \%)$. Hiperplasia linfóide (Figura 1) foi observada em 10 dos 24 apêndices $(41,7 \%)$ e eosinofilia em 13 (54,2\%). Evidências histológicas de invasão da mucosa

Departamento de ${ }^{1}$ Parasitologia, Instituto de Biociências, Universidade Estadual Paulista - UNESP, Botucatu, SP; Departamentos de ${ }^{2}$ Patologia e de ${ }^{3} \mathrm{Cirurgia}$ e Ortopedia Faculdade de Medicina, UNESP, Botucatu, SP.

Correspondência: Dra. Maria Aparecida M. Rodrigues - Departamento de Patologia, Faculdade de Medicina de Botucatu, UNESP - 18618970 - Rubião Júnior, Botucatu SP. E-mail:mariar@fmb.unesp.br 
do apêndice pelo E. vermicularis foram identificadas em quatro casos. Todos os pacientes receberam tratamento anti-helmíntico após a cirurgia e permaneceram assintomáticos durante o acompanhamento em ambulatório.

TABELA 1. Dados demográficos dos pacientes com apendicite parasitária

\begin{tabular}{|c|c|c|c|}
\hline \multirow{2}{*}{ Idade } & \multicolumn{2}{|c|}{ Sexo } & \multirow{2}{*}{ Total } \\
\hline & Masculino & Feminino & \\
\hline$<5$ & 3 & 2 & 5 \\
\hline $5-10$ & 7 & 4 & 11 \\
\hline $11-15$ & 3 & 0 & 3 \\
\hline $16-20$ & 1 & 0 & 1 \\
\hline$>20$ & 1 & 3 & 4 \\
\hline Total & 15 & 9 & 24 \\
\hline
\end{tabular}

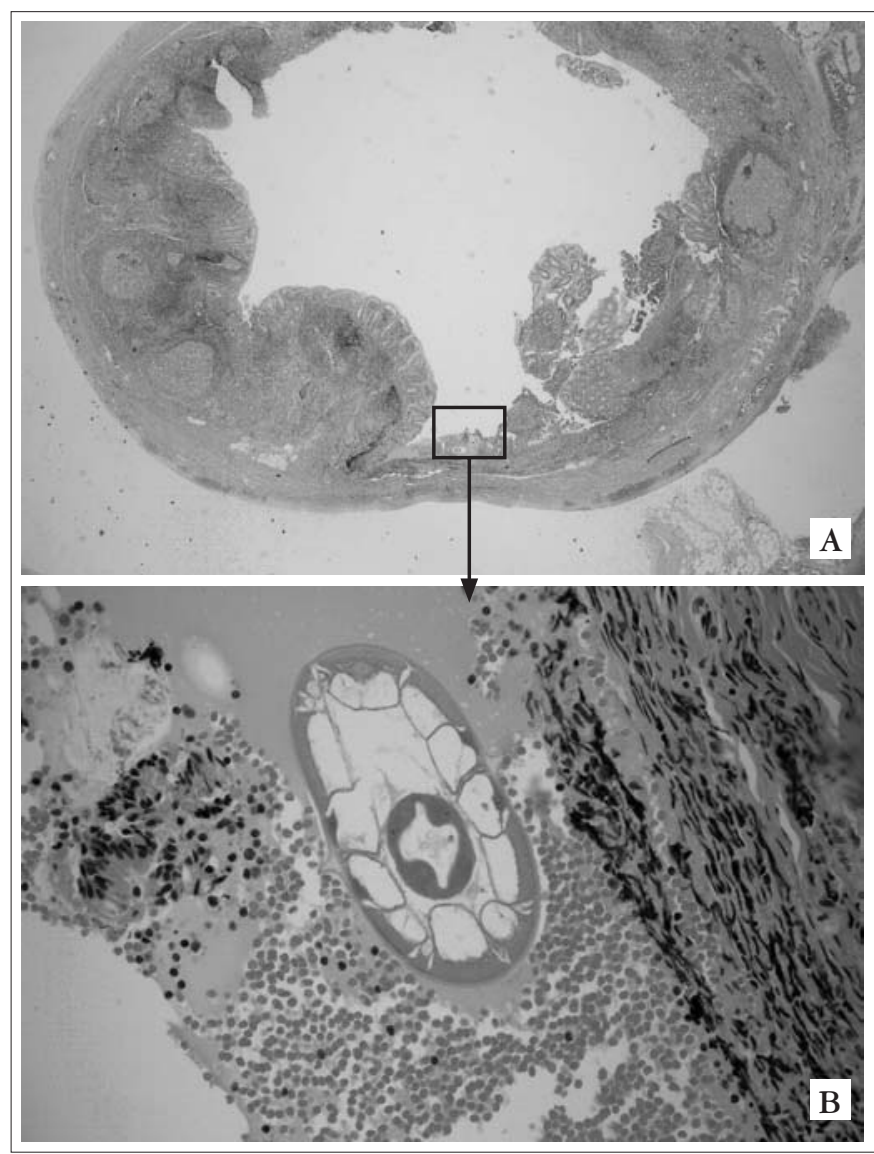

FIGURA 1. A) Fotomicrografia panorâmica do apêndice cecal associada à presença de Enterobius vermicularis na mucosa do órgão e hiperplasia linfóide. H-E. 25x. B) Detalhe da fotomicrografia anterior, evidenciando a região anterior do helminto. H-E. 200x

\section{DISCUSSÃO}

Os resultados do presente estudo demonstraram que parasitas são raramente encontrados no apêndice cecal e sua presença pode associar-se a sinais clínicos de apendicite. Estes dados documentam a eficácia das medidas sanitárias implementadas no Estado de São Paulo para o controle das infecções parasitárias intestinais.

Na presente série o E. vermicularis foi o parasita encontrado com maior freqüência no apêndice cecal. Este dado destaca a distribuição universal e o caráter endêmico da infecção por ele ${ }^{(1,3,5)}$. Por outro lado, a proximidade do apêndice ao habitat do Enterobius, que é a região do ceco, pode explicar o predomínio da infestação por este helminto no apêndice cecal.

Na presente casuística, tal infecção ocorreu com maior freqüência em crianças brancas menores de 10 anos, que constitui a faixa etária com maior prevalência de infecção por $E$. vermicularis ${ }^{(1,3)}$. Identificaram-se proglotes de Taenia sp. na luz do apêndice somente em uma paciente de 28 anos com quadro clínico de apendicite. $\mathrm{O}$ comprometimento do apêndice cecal por Taenia é raro, bem como tal apresentação clínica atípica de cólica apendicular ${ }^{(4)}$.

A associação entre infecção parasitária do apêndice cecal e apendicite aguda tem sido amplamente investigada. STILL ${ }^{(5)}$ foi o primeiro que relatou, em 1899 na Inglaterra, incidência de 19\% de infestação do apêndice por $E$. vermicularis em crianças com apendicite. A partir de então, inúmeros relatos têm apresentado informações contraditórias. A presença do parasita no apêndice pode levar a sinais e sintomas de cólica apendicular, independentemente do achado de inflamação ao exame histopatológico ${ }^{(1,3)}$.

Identificou-se no presente estudo amplo espectro de alterações patológicas do apêndice cecal em resposta à presença do $E$. vermicularis ou da Taenia $\mathrm{sp}$. As alterações morfológicas variaram de hiperplasia linfóide, até complicações potencialmente fatais, como gangrena e peritonite. Esta ampla variedade de alterações patológicas pode estar relacionada à evolução temporal da doença até o momento do diagnóstico e da intervenção cirúrgica. A hiperplasia linfóide, reacional aos antígenos do parasita, pode ser a resposta orgânica inicial responsável pelo quadro clínico de cólica apendicular. Por outro lado, os efeitos obstrutivos do tecido linfóide proeminente podem ser o fator desencadeante do processo inflamatório no apêndice cecal ${ }^{(2)}$. A inflamação aguda flegmonosa na muscularis própria, que constitui o atributo padrão-ouro para o diagnóstico histopatológico de apendicite ${ }^{(2)}$ foi encontrada na metade dos pacientes aqui estudados. Os resultados desta casuística compatibilizam-se com os de ARCA et al. ${ }^{(1)}$ que relataram alterações patológicas do apêndice na maioria das crianças com infestação por E. vermicularis. Ausência de inflamação histológica do apêndice na presença do parasita ocorreu somente em dois casos e pode estar relacionada à amostragem restrita do apêndice para o exame histopatológico.

Em síntese, os resultados do presente estudo demonstraram que infecções parasitárias do apêndice cecal são causa incomum de apendicite aguda em crianças e adolescentes. Enterobius vermicularis foi o helminto encontrado com maior freqüência na presente série e sua presença correlacionou-se com alterações patológicas do apêndice cecal, que variaram de inflamação aguda supurativa, hiperplasia linfóide e eosinofilia até complicações, como gangrena e peritonite. A possibilidade de infecção parasitária do apêndice cecal, apesar de ser pouco freqüente, deve ser considerada no diagnóstico diferencial da apendicite aguda, visto que o tratamento anti-helmíntico adequado é mandatório para solucionar o processo patológico básico e evitar complicações potencialmente fatais. 
Silva DF, Silva RJ, Silva MG, Sartorelli AC, Takegawa BK, Rodrigues MAM. Parasitic infection of the appendix and its possible relationship to acute appendicitis. Arq Gastroenterol. 2008;45(2):166-8.

ABSTRACT - From 1,600 surgically removed appendices, 24 (1.5\%) were found to have helminths. Enterobius vermicularis was observed in 23 of the 24 specimens (95.8\%) and Taenia sp was detected in only 1 (4.2\%) case. Sixteen patients (66.7\%) were less than 10 year-old; 15 patients were male and 9 female. Pathologic analysis disclosed acute neutrophilic inflammation in 12 cases and lymphoid hyperplasia in 10 of the 24 appendices. Gangrenous appendicitis was diagnosed in 3 cases and peritonitis was found in 11 of the 24 infested appendices. Parasitic infection of the appendix is an uncommon cause of acute appendicitis in children and adolescents.

HEADINGS - Appendix. Cecal diseases. Parasitic diseases. Appendicitis.

\section{REFERÊNCIAS}

1. Arca MJ, Gates RL, Groner JI, Hammond S, Caniano DA. Clinical manifestations of appendiceal pinworms in children: an institutional experience and a review of the literature. Pediatr Surg Int. 2004;20:372-5.

2. Lamps LW. Appendicitis and infections of the appendix. Semin Diagn Pathol 2004;21:86-97

3. Mowlavi GH, Massoud J, Mobedi I, Rezaian M, Solaymani MS, Mostoufi NE, Gharaguzlo MJ. Enterobius vermicularis: a controversial cause of appendicitis. Iranian J Publ Health. 2004;33:27-31.
4. Sartorelli AC, da Silva MG, Rodrigues MA, da Silva RJ. Appendiceal taeniasis presenting like acute appendicitis. Parasitol Res. 2005;97:171-2.

5. Still GF. Observation on Oxyuris vermicularis in children. Br Med J. 1899;1:898. 